Résumés des conférences et travaux

\title{
Histoire de l'art de la Renaissance
}

\section{Sabine Frommel}

\section{(2) OpenEdition Journals}

Édition électronique

URL : https://journals.openedition.org/ashp/3898

DOI : $10.4000 /$ ashp.3898

ISSN : 1969-6310

Éditeur

Publications de l'École Pratique des Hautes Études

\section{Édition imprimée}

Date de publication : 1 septembre 2020

Pagination : 277-279

ISSN : 0766-0677

\section{Référence électronique}

Sabine Frommel, "Histoire de l'art de la Renaissance », Annuaire de l'École pratique des hautes études (EPHE), Section des sciences historiques et philologiques [En ligne], 151 | 2020, mis en ligne le 09 juillet 2020, consulté le 06 juillet 2021. URL : http://journals.openedition.org/ashp/3898 ; DOI : https:// doi.org/10.4000/ashp.3898 


\title{
HISTOIRE DE L'ART DE LA RENAISSANCE
}

\author{
Directrice d'études : M ${ }^{\text {me }}$ Sabine Frommel
}

Programme de l'année 2018-2019: La pensée architecturale de Léonard et sa fortune au $X V I^{e}$ siècle.

Peu étudiée, la production de dessins d'architecture du Florentin a été considérée jusqu'à présent comme un phénomène isolé, dépourvu de tout parallèle avec les inventions de ses contemporains et de toute postérité. Après avoir approfondi pendant l'année universitaire 2018-2019 les réflexions de Léonard dans ce champ et l'évolution de celles-ci, en procédant selon des typologies architecturales, nous nous sommes ensuite consacrés aux références qu'il a élaborées, aux contaminations et aux influences de ses projets, en grand partie idéaux, sur d'autres maîtres. Étant donné les rares documents écrits, susceptibles d'éclaircir ce problème, nous nous sommes appuyés sur un corpus de dessins concernant l'architecture religieuse, publique et privée. De cette manière s'est dégagé un réseau de contacts qui permet de mieux situer l'œuvre de Léonard dans le développement des typologies et des langages architecturaux à la fin du Quattrocento et au début du Cinquecento.

Avant son départ pour la cour des Sforza à Milan en 1482, Léonard assista au renouveau dans le domaine de l'art de bâtir, promu par Laurent de Médicis, sous l'influence de l'œuvre théorique et pratique de Leon Battista Alberti. L'église à plan centré et la «sacralisation » de la demeure, également centrée et dotée de serliennes ou de façade de temple, devaient l'inspirer tout au long de sa carrière. L'Adoration des Mages (Florence, Offices), le seul tableau que Léonard dota d'une ruine à l'antique et développa dans plusieurs variations, reflète clairement cette influence. Une rencontre avec Francesco di Giorgio Martini à Milan et à Pavie en 1490 lui permit de prendre connaissance de ses investigations autour des fortifications, ce qui a été confirmé par la recherche, mais de nombreux dessins et croquis attestent que l'architecte-ingénieur siennois lui donna aussi des témoignages graphiques de ses études de palais (Cod. Magliabechiano), fondées sur des systèmes géométriques rationnels. En 1492, Léonard rencontra Giuliano da Sangallo à Vigevano, qui présenta à la cour des Sforza une maquette en bois de la villa Médicis de Poggio à Caiano et ses projets idéaux à partir de ce moment attestent en toute netteté l'impact de cette demeure.

Si l'échange intense avec Bramante qui s'était établi à la cour des Sforza entre 1482 et 1499 a suscité l'intérêt des chercheurs, on ne s'était pas posé la question d'une poursuite de ce dialogue pendant les séjours du Florentin à Rome en 1501 et 1505. Le premier est documenté par une notice sur une feuille du Codex Atlanticus (227r-a [618v]) qui se réfère à une visite à la Villa Hadriana à Tivoli, suivi sans doute par une autre dans la Ville éternelle - des expériences qu'il aurait pu entreprendre avec Bramante. Afin de se hisser au rang d'excellent connaisseur de l'héritage classique, ce dernier menait des enquêtes archéologiques qui encouragèrent de nouvelles méthodes d'assimilation des prototypes classiques, dont il fit preuve avec le tempietto de San Pietro in Montorio. Bien évidemment, Léonard fut attentif aussi 
à d'autres arts comme la sculpture et la Pietà du jeune Michel-Ange à Saint-Pierre semble avoir inspiré la posture de son carton pour la Sainte Anne, à laquelle il devait se consacrer après son retour à Florence. Un document de douane du 30 avril 1505 semble confirmer qu'il fut de nouveau dans la cité pontificale, précisément lorsque le débat autour du tombeau du Jules II et de la reconstruction de Saint-Pierre atteignit son apogée. Ses recherches dans le domaine de l'église à plan centré, le seul corpus cohérent relatif à cette typologie pendant cette période, le recommandèrent pour une intervention à ce moment crucial, sollicité peut-être par Bramante. Le plan centré de ce dernier (GDSU 1) que Jules II retint pour la basilique pétrinienne renvoie directement à certains croquis conçus pendant la période milanaise, issus sans doute d'une réflexion partagée. L'influence du tombeau isolé dessiné par Michel-Ange est sensible dans le projet de Léonard pour la sépulture de Gian Giacomo Trivulzio, conçue pendant son deuxième séjour à Milan (Windsor, Royal Library RL 12355r).

Rome fournit un important creuset de références qui allaient changer le vocabulaire de Léonard : les travées rythmiques de la cour supérieure du Belvédère; la combinaison de l'attique et du fronton, archéologiquement erronée, en couronnement des façades, proposée par son compatriote Giuliano da Sangallo. Lorsqu'il dessina vers 1506 son projet pour la villa pour Charles d'Amboise près de Milan (Cod. Atl. f. 231v-a [628r]), il se rappela le dessin de Baldassarre Peruzzi pour la Farnésine, la spectaculaire demeure extra muros du banquier Agostino Chigi, conçue précisément en 1505. Des projets d'Antonio da Sangallo le Jeune et des dessins du Codex Chlumczansky (Prague, bibliothèque du Musée national), attribuable à un artiste de l'entourage de Giulio Romano, révèlent que ce dessin n'était pas inconnu; au début des années 1530, Baldassarre Peruzzi devait copier une variante à plan centré de cette villa, regroupée sur une même feuille à côté de la version définitive.

Parmi les liens et les contacts qui influencèrent les recherches de Léonard, les Florentins occupèrent une place d'exception, de Giuliano da Sangallo et son frère cadet Antonio il Vecchio, leur neveu Antonio il Giovane jusqu'à Michel-Ange. Un croquis d'Antonio da Sangallo le Jeune porte à croire que ce dernier a vu aussi le projet de Léonard pour la restructuration de la place devant l'église Saint-Laurent, mausolée des Médicis (Codex Atlanticus, f. 315r-b [865r]). Comme d'autres artistes, Léonard avait été sollicité pour des dessins concernant l'entrée triomphale de Léon X à Florence en décembre 1515 et l'essor du retour de la dynastie dans la capitale toscane aurait pu le motiver à imaginer une telle intervention. Bien que les contacts avec Buonarroti fussent tendus, une influence réciproque se dégage avec des contours assez clairs. $\mathrm{Si}$ le carton de la Sainte Anne renvoie à la Pietà du jeune Michel-Ange, ce dernier copia et varia en 1501 ce même carton qui devait le conduire à des représentations plus complexes de figures. Pendant son dernier séjour à Rome de 1513 à 1516, Léonard réussit à le convaincre de tourner la tête du Moïse (tombeau de Jules II, changement effectué en 1534), afin d'accentuer la plasticité de la figure. Lorsqu'il conçut le portail de la bibliothèque Laurentienne, marqué par un emboîtement de deux frontons, il aurait pu se souvenir d'un dessin de Léonard qui avait entrepris des recherches similaires.

Force est de constater que le Florentin développa ses projets par le biais d'intenses échanges avec d'autres artistes et sut élaborer de manière originale de multiples références. Sans jamais construire un monument, il contribua à promouvoir l'évolution 
des typologies architecturales, alors qu'il restait indifférent au vocabulaire architectural et à la mise au point d'un langage normatif d'après les prototypes de l'Antiquité romaine et le traité de Vitruve. Par cette attitude, Léonard tranche avec les préoccupations des architectes de son temps et montre que sa vision de cet art gravite autour de la fonctionnalité, de la tridimensionnalité et de la clarté géométrique.

Les conférences ont été suivies par 15 doctorants (en partie co-tutelle), trois " post-doctorants », sept étudiants en master 1 et 2, cinq étudiants Erasmus et environ 20 auditeurs. Elles ont été accompagnées de séminaires destinés à la présentation et la discussion des travaux des étudiants de master 2 et des doctorants. 